\title{
L'ACADÉMIE VÉTÉRINAIRE DE FRANCE ET SA CONTRIBUTION AUX ENJEUX ACTUELS DE LA POLITIQUE SANITAIRE
}

\author{
THE FRENCH VETERINARY ACADEMY AND ITS CONTRIBUTION TO \\ CURRENT HEALTH POLICY ISSUES
}

Par Julien DENORMANDIE ${ }^{1}$

(Discours de clôture du Ministre de l'agriculture et de l'alimentation, Séance solennelle de l'Académie vétérinaire de France du 3 décembre 2020)

Mots-clés : Académie vétérinaire de France, Une seule santé, bien-être animal, vétérinaires

Key words : French veterinary Academy, One Health, animal welfare, veterinarians

Monsieur le Président,

C'est vraiment avec un grand plaisir et beaucoup de reconnaissance que je m'adresse devant vous cet après-midi. C'est vrai que j'aurais préféré pouvoir être physiquement présent, dans le superbe amphithéâtre de l'Académie nationale de médecine mais je crois que c'est aussi tout l'esprit d'innovation de l'Académie vétérinaire de France (AVF) que de pouvoir tenir tout de même cette séance solennelle en dépit des contraintes du Covid.

Je voudrais d'abord commencer mes propos par vous remercier Monsieur le Président Angot, pour l'action que vous menez à la tête de cette Académie et par votre truchement de l'ensemble des membres de l'AVF. C'est sûr que pour l'Académie, ce moment est important. C'est une opportunité, vous l'avez dit, de pouvoir échanger avec des illustres experts. Vous avez parlé de mon ami Érik Orsenna ou du professeur Gilles Boeuf. Je fais miens les propos que vous avez tenus : il est important dans la période également de donner la perspective de pouvoir s'inscrire dans le temps long et de pouvoir également voir comment dans la crise que nous vivons, les opportunités qu'il serait possible de développer. Et puis, plus fondamentalement pour moi, c'est une occasion de remercier l'Académie pour les différents travaux qu'elle conduit, tout au long de l'année. Je crois vraiment que votre rôle est important. Je ne le dis pas parce que je suis devant vous cet après-midi. J'en suis convaincu. Votre rôle est important et il est d'autant plus important je crois pouvoir le dire dans la période que nous vivons. D'abord parce que ce qui fait l'ADN de l'Académie c'est avant tout la Science et le Progrès. Et ces notions sont parfois mises à mal actuellement dans notre société : une société de l'émotion, une société des réseaux sociaux, une société du court-terme. La Science est parfois décriée, la Science est parfois considérée comme quelque chose qu'elle n'est pas ou bien elle est remise en cause. Elle serait même parfois balayée d'un revers de main par certains qui détiendraient une vérité non fondée sur la Science. Et cela emporte évidemment une société et surtout une société qui doit être dirigée par ce progrès. J'en suis d'autant plus convaincu que je fais partie de ceux qui considèrent que la politique ne peut jamais être une politique de l'émotion. Cela ne doit jamais être non plus une politique de la vertu. La vertu n'est pas l'essence de la politique. Cela doit être une politique de la raison. Et la raison est parfois oubliée exactement pour les mêmes raisons que j'évoquais à l'instant. Le fait d'avoir la science et le progrès et des institutions qui les mettent en avant, qui font vivre cela, c'est à mes yeux important et cela fait partie de ces piliers des politiques publiques, de cet engagement républicain que l'actualité ne cesse de mettre en avant comme des éléments encore plus nécessaires aujourd'hui qu'ils ne l'étaient hier. Donc, dans ce contexte incroyablement difficile, qui est aussi singulier que difficile, je crois qu'à la fois, la vision, la stabilité, la valeur ajoutée d'institutions comme l'Académie vétérinaire est quelque chose d'essentiel pour le Ministre des vétérinaires que je suis et le Président d'honneur de l'Académie que je suis également. Je crois pouvoir dire d'ailleurs, que vous avez fait preuve de réactivité. Je citerai un seul exemple : vous avez tout de suite mis en place une cellule Covid 19, regroupant

1. Ministre de l'Agriculture et de I'Alimentation, 251 rue de Vaugirard, 75732 Paris cedex 15 
l'ensemble des compétences pour vous demander comment l'Académie pouvait apporter sa pierre à l'édifice, sa contribution aux débats, sa valeur ajoutée aux travaux. C'est tout à votre honneur d'avoir tout de suite décidé et de manière très précoce cette mise en place et au final, de se dire, comment la science et notamment le savoir-faire vétérinaire étaient des éléments très importants dans le débat actuel. Je le dis vraiment avec beaucoup de conviction et c'est constitutif pour moi, de l'engagement politique au sens noble du terme : ce qui fait la politique d'une nation, la politique de la Cité, la Cité au sens grec du terme. Au-delà de ces éléments introductifs, je voudrais en tant que Ministre des vétérinaires, insister sur trois sujets cet après-midi devant vous. Trois sujets qui me paraissent non seulement importants mais qui résument assez bien, je crois, les principaux enjeux qui doivent nous guider collégialement dans les prochains mois et les prochaines années. Ces trois sujets sont : le concept «Une seule santé », le «bien-être animal » et le " maillage vétérinaire ».

\section{ONE HEALTH / UNE SEULE SANTÉ}

Les vétérinaires jouent un rôle central pour relever ce défi d'" Une seule santé ". Nos politiques publiques doivent s'intégrer complètement dans le concept "One health " pour mieux comprendre et réagir face aux risques de maladies zoonotiques pouvant devenir pandémiques. Lutter contre l'émergence ou la diffusion de ces pandémies, c'est prendre en compte les interactions entre modifications de l'écosystème, le changement climatique, les déséquilibres des organismes vivants (micro-organismes, vecteurs, réservoirs et hôtes) et les activités humaines. Au niveau international, cette approche interdisciplinaire prend forme à travers la création récente d'un Conseil d'experts de haut niveau "One health ", qui vient d'être annoncée dans le cadre du Forum de Paris pour la paix le 12 novembre 2020. Je suis convaincu des vertus d'une telle approche «One health». Et les vétérinaires sont et doivent rester des acteurs actifs de sa mise en œuvre. De solides coopérations interministérielles existent déjà avec le Ministère des solidarités et de la santé notamment sur la gestion des aliments contaminés et des toxi-infections alimentaires afférentes, sur la réduction de l'utilisation des antibiotiques. La présence conjointe aujourd'hui de deux ministres en est encore un exemple, preuve s'il en fallait du lien entre agriculture, santé animale et biodiversité. Je sais par ailleurs que Bérangère Abba (secrétaire d'État chargée de la biodiversité) a récemment commandé des avis à l'Académie vétérinaire sur des problématiques relatives à la faune sauvage. Nous travaillons également en interministériel sur la gestion globale des risques sanitaires, notamment dans la prévention des zoonoses issues de la faune sauvage. La nouvelle édition du Programme national santé-environnement, le 4ème, sera bientôt finalisée et va dans ce sens. Je le dis souvent : « il faut toujours mieux prévenir pour avoir moins souvent à guérir ». En cela le travail de l'Académie vétérinaire fondée sur la concertation avec les parties prenantes (agences et instituts performants, platefomes d'épidémio-surveillance, acteurs de terrain mobilisés, Académie de médecine...) est précieux. Les vétérinaires dans leur diversité se sont largement mobilisés pour la santé de nos concitoyens lors de cette crise Covid, en particulier au printemps pour mettre à disposition des médecins du matériel, pour réaliser des analyses PCR. Je renouvelle aujourd'hui mes remerciements à leur égard. Le premier des enjeux pour les décennies à venir, et vous l'avez dit, Monsieur le Président et j'en ai l'immense conviction, est la mise en place d'une politique "One health ". Cette politique "One health», certains précurseurs, visionnaires, l'ont initié maintenant depuis de nombreuses années. Elle n'a jamais été autant d'actualité qu'aujourd'hui. De nombreuses études ont montré que $60 \%$ des maladies humaines infectieuses aujourd'hui étaient d'origine animale, que des maladies émergentes impliquent la faune sauvage. Mais cette stratégie "One health ", au-delà de ces études, rappelle à toutes et tous que l'Homme (au sens espèce humaine) est un maillon d'un écosystème, un maillon d'une chaîne du vivant et donc qu'il n'est pas indépendant de cet écosystème ni de cette chaîne du vivant. Et encore une fois, dans les politiques publiques mises en place, cette approche holistique qui emporte à la fois la nécessité d'une vision complexe et en même temps l'humilité de la place de l'Homme dans un écosystème est fondamentale. C'est tout aussi révolutionnaire que fondamental d'ailleurs car, il est beaucoup plus facile de courir chacun dans son couloir ou de nager dans son couloir de nage plutôt que d'avoir cette vision holistique pour répondre à un problème complexe. Je pense, je crois pouvoir dire, qu'il y a quelque chose qui est en train de se passer sur cette vision "One health ». Aujourd'hui on le voit bien, cette politique d'Une seule santé est beaucoup plus évoquée ou en tout cas mise en œuvre dans les politiques, à l'échelle nationale, européenne et internationale qu'elle ne l'était jusqu'à présent. Mais il y a encore beaucoup de choses qu'il nous faut faire. Je vous donne un certain nombre d'exemples. Dans le cadre de la crise Covid on voit bien que l'approche vétérinaire est un élément essentiel et de compréhension et de gestion. Compréhension quant à l'origine, gestion quand vous voyez ce qui se passe avec les visons en France et surtout dans d'autres pays ; mais aussi d'accompagnement au jour le jour de la gestion de l'épidémie. Pour moi, c'est une conviction chevillée au corps, les vétérinaires ont un rôle très important à jouer au moment de l'apparition d'une épidémie. Mais la gestion d'une épidémie doit donner place aujourd'hui à la prévention de ces épidémies dans le futur. Il vaut évidemment prévenir que guérir mais prévenir nécessite d'avoir une approche holistique et cette approche "One health ». Je pense qu'il y a quelque chose qui est en train de se passer en la matière mais je pense en même temps qu'il y a encore beaucoup de chemin à parcourir. La création au niveau mondial d'un Conseil d'experts de haut niveau "One health " est une excellente nouvelle. On a encore toutefois beaucoup de chemin à parcourir : c'est une initiative diplomatique plus que sanitaire. C'est une initiative discutée au niveau du Ministère des affaires étrangères, ce qui est très bien mais on doit passer demain à une véritable approche en termes de politique publique sanitaire sectorielle et de gestion. Je fais partie de ceux qui sont toujours extrêmement étonnés en amont de chacun des remaniements ministériels par le débat sur l'éventuel rattachement du Ministère de l'agriculture et de l'alimentation (et donc des vétérinaires) au Ministère de l'environnement ou vice-versa. J'ai toujours été très surpris de constater que le vrai débat sur le travail conjoint avec le 
Ministère de la santé n'était jamais mis en avant. La première des missions et la première des actions et la première des préventions que ce ministère doit avoir c'est l'approche de la santé. Evidement le volet environnemental est d l'un des éléments de cette santé mais cela emporte aussi le volet nutritionnel et plus globalement l'approche Une seule santé. J'ai beaucoup d'échanges avec le Ministère de la santé au jour le jour. Je crois qu'il y a de solides coopérations interministérielles qui commencent à naître, à se développer. Citons l'exemple du programme Ecoantibio, où l'on voit les avancées que l'on a réussi à obtenir dans le cadre des coopérations interministérielles. J'ai une vision politique qui est très claire. Il nous faut, à la fois favoriser, consolider le "One health " mais aller plus loin, tirer profit du moment que nous vivons en tant qu'une opportunité pour inscrire dans la durée cette politique holistique de santé et considérer ce le paradigme "One health " comme quelque chose qui guide notre action. De manière très concrète, j'ai souhaité que la DGAL, qui est de facto au sein du Ministère positionnée entre les préoccupations de santé animale, végétale, humaine et environnementale, incarne véritablement cette politique "One health " au travers de ses priorités d'action et même de son organisation. C'est quelque chose qui est à mes yeux d'autant plus important que nous aurons face à nous, un travail très important au lendemain de la sortie de la Covid 19, sur la prévention des zoonoses et la nouvelle édition du Programme Santé-Environnement. Je remercie à la fois l'Académie vétérinaire mais aussi les services du Ministère.

\section{BIEN-ÊTRE ANIMAL}

La deuxième conviction que je voulais partager avec vous est celle du rôle de notre action vis-à-vis de transitions auxquelles est confronté notre pays, transitions qui, souvent sont amplifiées par la demande sociétale. Je voudrais prendre l'exemple du bien-être animal (BEA). Le sujet du BEA est absolument fondamental. Je ne connais personne qui soit contre le BEA. La question du BEA c'est : comment on arrive à répondre à une demande sociétale de plus en plus forte tout en accompagnant celles et ceux à qui nous donnons cette injonction de plus de BEA? Nous vivons aujourd'hui dans un pays où la préoccupation du BEA est parmi les plus fortes en Europe. Je le constate avec tous mes collègues ministres de l'agriculture d'autres pays européens. Pourtant, nous faisons partie des pays qui détiennent quelques tristes records. Je pense à l'abandon des animaux domestiques, pour ne citer que lui. On a, face à nous, une réconciliation à avoir entre la demande de plus de BEA et un comportement collectif associé à ce sujet. Il y a plusieurs sujets très importants qu'il nous faut aborder. D'abord le sujet de la maltraitance animale et je le dis très clairement, le sujet du BEA est souvent mis à mal par un amalgame stérile entre maltraitance animale et BEA. Ce sont évidemment deux choses très différentes. La maltraitance est d'ailleurs pénalement répréhensible y compris avec des peines de prison. Et la maltraitance résulte d'un acte délibéré de nuire à un animal. Le débat, on le voit tous les jours, fait un amalgame énorme entre la maltraitance et le BEA. Dans un abattoir on parle de bien-être animal même si on sait que l'issue, pour un animal qui rentre à l'abattoir n'est pas une issue heureuse. La maltraitance doit être combattue avec la plus grande fermeté. Jamais notre main ne doit trembler vis-à-vis d'actes de maltraitance, que cela soit la maltraitance des animaux domestiques avec notamment la question des animaux abandonnés ou que ce soit la maltraitance des animaux d'élevage. Dans les deux cas, sur le sujet du BEA, il nous faut accompagner la société. Vous demandez à n'importe qui s'il préfère qu'une poule soit élevée en cage ou en plein air, il vous répond évidemment "je préfère que la poule soit élevée en plein air ". Et au même moment vous demandez " est-ce que vous acceptez d'avoir près de 80 millions de poules lâchées en liberté ?", avec les risques sanitaires associés, qui plus est dans un contexte de l'influenza aviaire, et les conséquences que cela peut avoir y compris sur le coût, et on vous répond «non, ce serait de la folie ". Donc il nous faut, dans un débat qui est passionné et avec beaucoup de sincérité de part et d'autre, remettre, là aussi, une forme de raison. Je compte beaucoup sur vous et vous pourrez compter sur moi pour que ce volet de raison puisse être au cœur des décisions à prendre sur ces sujets. Érik Orsenna a évoqué avec vous le sujet. Quand vous lisez son dernier ouvrage "Voyage aux pays du cochon ", tout cela transparaît de manière extrêmement précise. Je pense que les vétérinaires, ont un rôle majeur en mettant à chaque fois la raison au centre de tout. J'évoquais la lutte contre l'influenza aviaire mais on pourrait dire la même chose avec les cochons sur la nécessité d'avoir des formes de protection de nos élevages, vis-à-vis de la peste porcine africaine ou d'autres maladies. Mais au-delà de cela, vous avez aussi un rôle à jouer en tant qu'acteurs du quotidien, dans les exploitations, dans les élevages, dans les refuges, comme quelqu'un qui conseille, qui accompagne, qui soigne - c'est le cour de votre métier - et qui alerte. Dans les prochaines semaines, les prochains mois, nous allons, au sein du ministère, mettre à jour la stratégie du ministère sur le BEA. Il faut que l'on puisse travailler conjointement avec vous sur ce sujet. L'objectif est de faire cela au début de l'année prochaine ; je sais que l'Académie vétérinaire a réalisé des travaux sur le sujet et je compte bien pouvoir m'en inspirer.

\section{MAILLAGE VÉTÉRINAIRE}

Enfin, le troisième sujet que je voulais évoquer avec vous est la question du maillage vétérinaire. C'est pour moi un gros sujet de préoccupation. Il l'est pour beaucoup d'acteurs du territoire, beaucoup d'élus et notamment beaucoup de parlementaires. Avec cette question du maillage territorial, nous sommes aujourd'hui confrontés non pas à des déserts médicaux mais à des déserts vétérinaires. C'est un gros sujet de préoccupation que l'on voit de plus en plus apparaître. On va avoir une idée plus précise de la réalité grâce au recensement agricole que nous venons de lancer, en obtenant des données précises sur le nombre d'élevages, le nombre d'animaux quel que soit le type d'élevage et l'on va pouvoir donc avoir une cartographie encore plus précise que celle que nous avons les manques et donc sur les déserts vétérinaires. Le maillage renvoie à plusieurs sujets. Il renvoie évidemment à l'attractivité du métier, qui est un sujet que vous suivez évidemment. Cela renvoie à la question de l'installation de vétérinaires, que ce soit pour les animaux domestiques ou pour les animaux de rente. Vous savez qu'on a, depuis le début des années 2010, essayé de renforcer la 
formation en accroissant le nombre d'élèves dans les écoles vétérinaires (+ 35\%). Dans l'enseignement, il y a beaucoup de travail d'accompagnement des élèves vis-à-vis de la profession vétérinaire. Je pense par exemple aux stages qu'on peut offrir à nos élèves dans les lycées agricoles où l'on voit bien, pour avoir échangé avec un grand nombre d'élèves depuis que je suis à la tête de ce ministère, à quel point le rôle des stages et du parcours éducatif est important pour susciter et accompagner les vocations dans les métiers relatifs aux animaux domestiques mais également dans les métiers de l'élevage. Le deuxième volet d'attractivité ce sont toutes les dispositions que nous avons prises très récemment dans la loi DDADUE. Elle vient d'être signée par les différents ministres. Elle permet d'apporter un certain nombre d'aides comme les aides aux installations, que nous pouvons faire dorénavant en lien avec les collectivités locales, elles-mêmes désireuses de pouvoir aider des installations y compris d'un point de vue financier. Le troisième volet est, au-delà du parcours de formation et de l'aide à l'installation, le nombre d'élèves qu'on arrive à former. On sait aujourd'hui que, sur la période 2019, la France aura besoin de plus de 1000 vétérinaires nouveaux par an, ce qui est colossal. Dans le cadre du Brexit nous sommes en train de mettre en place des contrôles sanitaires qui impliquent énormément le réseau vétérinaire et nous constatons l'incapacité ou l'immense difficul té qu'on a à recruter à tel point que nous devons embaucher un certain nombre de vétérinaires étrangers. Je ne parle pas non plus des formations à l'extérieur et donc face à cela nous avons deux enjeux. Le premier est d'essayer d'accroître les capacités d'accueil des écoles actuelles. Je disais que depuis 2012 elles ont augmenté de $35 \%$. Est-ce qu'on peut aller plus loin dans les écoles actuelles ? En tout cas, à mon avis, il faut vraiment continuer à consolider les quatre écoles nationales vétérinaires. Et le second, est le sujet de nouvelles écoles, sur lequel je pense qu'il faut avancer. J'ai suivi comme vous tous les débats qui ont lieu en ce moment sur les décisions votées au Parlement sur l'ouverture d'écoles dites à statut privé, mais je le précise, conventionnées avec le ministère de l'Agriculture et à but non lucratif. Nous avons un manque important de vétérinaires et il nous faut former des vétérinaires. Il nous faut continuer à avancer je le pense sur ce sujet-là en dépit des critiques qui peuvent être émises ici ou là. Enfin, je vous informe que j'ai signé hier le décret sur le recrutement post-bac, mesure qui est tout à l'honneur de la profession. C'est un long travail qui va permettre dès cette année d'ouvrir 160 places à travers Parcoursup, donnant aussi sa chance à des élèves qui parfois subissent une barrière dans l'accès aux classes préparatoires. Je voudrais saluer tout le travail réalisé par la profession pour permettre cela.

\section{CONCLUSION}

Mesdames et Messieurs, voilà ce que je voulais partager avec vous. Je vous adresse mes remerciements concernant les travaux que vous menez et l'approche qui est la vôtre. Il est à mes yeux extrêmement important que Raison et Science puissent être mis au centre des débats sans jamais céder à l'émotion et à la force du temps court. Je crois que c'est essentiel pour une bonne politique et trop souvent oublié. En tout cas, pouvez être sûrs que c'est ce qui guide mon action au jour le jour.

Les défis sont immenses. Celui du "One health » est absolument primordial car il va nous confronter au jour le jour à la constitution de l'écosystème dans lequel nous vivons. Les sujets de société, comme le bien-être animal, doivent pouvoir être appréhendés avec beaucoup de force et de conviction sans craindre les débats. Enfin, les sujets de l'exercice du beau métier de vétérinaire, du maillage territorial et donc de l'accroissement des formations sont à mes yeux essentiels. Je sais que cela suscite des débats mais il nous faut les avoir avec la volonté de faire en sorte qu'il y ait encore plus de vétérinaires dans notre pays. La contribution vétérinaire n'est pas seulement scientifique et technique. Confronté aux réalités de la société et en particulier du monde agricole, attentif au bien-être humain et au bien-être animal, le vétérinaire joue un rôle majeur dans les domaines sociologique et économique.

Je vous remercie pour votre invitation à venir clore vos travaux et je compte sur vous. 